\title{
Solid State Photodecarbonylation of Diphenylcyclopropenone: A Quantum Chain Process Made Possible by Ultrafast Energy Transfer
}

\author{
Gregory Kuzmanich, Arunkumar Natarajan, Khin K. Chin, Marcel Veerman, Christopher J. Mortko, \\ and Miguel A. Garcia-Garibay* \\ Department of Chemistry and Biochemistry, University of California, Los Angeles, California 90024-1569
}

mgg@chem.ucla.edu

\section{Contents}

Page Numbers

1) Experiment Details and Purification of 1, 2, and $\mathbf{3}$

2) Determination of the Relative Absorbances of DPCP and DCK

3) Experimental Details for Quantum Yield Studies

4) Relative Absorbances of DPCP and DCK as a Function of Loading

5) Qualitative Analysis of the Reaction Efficiency as a Function of Excitation to $\mathrm{S}_{1}$ and $\mathrm{S}_{2}$

6) Experimental Evidence of a Solid-to-Solid Reaction by Fluorescence Analysis

7) Experimental Studies of Changes in Nanocrystal Size as a Function of Reaction Progress

8) Infrared Spectral Analysis of "as Formed" and Recrystallized DPA

9) Details on the Accompanying Time-Lapse Microphotographs 


\section{1) Experiment Details and purification of 1, 2, and 3}

General: All chemicals were purchased from Sigma Aldrich Co., Inc., and used without further purification, with the exception of diphenylcyclopropenone and diphenylacetylene, which were recrystallized prior to use. All suspensions were prepared immediately before irradiation. ${ }^{1} \mathrm{H}$ and ${ }^{13} \mathrm{C}$ NMR spectra were obtained with a Bruker Advance 300 spectrometer. IR spectra were obtained with a Perkin-Elmer Spectrum instrument equipped with a universal Attenuated Total Reflectance (ATR) accessory. Gas Chromatography data was acquired on a Hewlett-Packard 5890 Series II Gas Chromatography equipped with an HP3396 Series II integrator and HP-5 capillary column of dimension $25 \mathrm{~m} \times 0.2 \mathrm{~mm}$ with a film thickness of $0.11 \mathrm{~mm}$. The excitation and emission spectrum of all samples was obtained on a SPEX FLUOROLOG spectrofuorimeter. Dynamic Light Scattering measurements were taken on a Beckman-Coulter N4 Plus Submicron Particle Size Analyzer. Silica used for purification was Silia-P Flash Silica Gel (40-62 $\AA)$, purchased from SiliCycle Inc. UV-Vis spectra were taken on a Beckman DU-650 spectrometer.

Purification of Diphenylcyclopropenone (DPCP) (1): Diphenylcyclopropenone (98\%) was purchased from Sigma-Aldrich Co., Inc. DPCP was recrystallized from methylene chloride/water (8:2), and eluted with methylene chloride through a silica column. DPCP was then recrystallized slowly in cyclohexane to give pure white needles.

Purification of Diphenylacetylene (DPA) (2): Diphenylacetylene was recrystallized in cyclohexane prior to fluorescence studies, to give pure white flakes.

Preparation of Dicumyl ketone (DCK) (3): Dicumyl ketone was synthesized as previously reported [Resendiz, M. J. E.; Garcia-Garibay, M. A., Resendiz, Marino J. E.; Garcia-Garibay, Miguel A. Org. Lett. 2005, 7, 371-374.]. DCK was then recrystallized in a 1:1 mixture of ethyl acetate and acetone to give single crystalline prisms of pure DCK. 


\section{2) Determination of the Relative Absorbances of DPCP and DCK}

Equimolar suspensions of DCK and DPCP were made as described above. UV-Vis spectra of the two suspensions before and after mixing were acquired with a fiber optic immersion probe and plotted along with the reported emission spectrum of the RPR-3000 lamps. The results of these measurements confirmed that the spectrum of the mixed components is the average of the two independently obtained spectra. The relative absorbance of the two components was obtained by calculating the ratio of the spectral integral of the area that overlaps with the spectrum of the lamp. The result of this measurement and subsequent calculation was an absorbance ratio, $A_{D P C P} / A_{D C K}=4.88$. This value was used to calculate the relative absorbance of the two compounds by multiplying it by the relative ketone loading values.

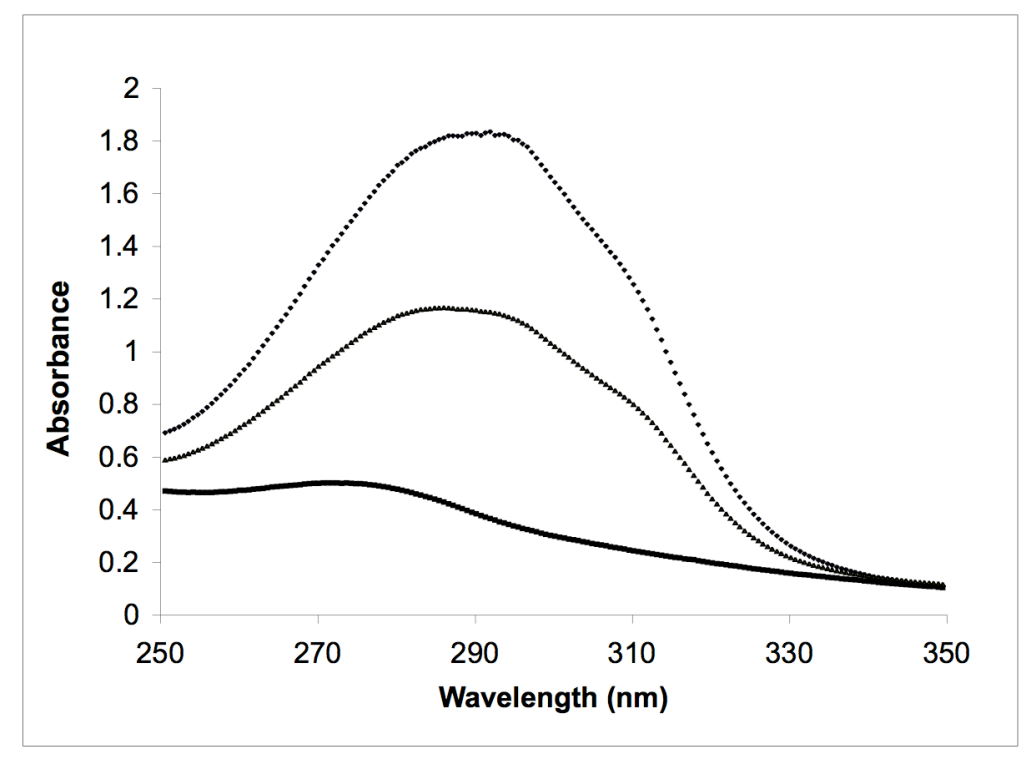

Figure S1: Absorption spectra of equimolar-loaded DPCP (top), DCK (bottom) and combined (middle) nanomolar aqueous suspensions. Spectra were measured with an immersion fiber optic probe.

\section{3) Experimental Details for Quantum Yield Studies}

General: All quantum yield studies were preformed in a Rayonet Photochemical Reactor using $312 \mathrm{~nm}$ lamps (BLE-8T312), and a Pyrex $(\lambda>290 \mathrm{~nm})$ filter. All filtered photochemical reactions were carried out using a 450W medium pressure Ace - Hanovia mercury arc lamp (Catalog No. 7825-34), equipped with a circulating water cooled Pyrex jacket. 
Surfactant Solutions: Solutions of surfactant in water were made at $1 / 20^{\text {th }}$ of the critical micelle concentration (CMC) for both CTAB and SDS in water. Micelar media was prepared with $200 \%$ CMC levels of CTAB in water

Quantum Yield Determination: Optically dense suspensions of DPCP and DCK were prepared by the reprecipitation method [Kasai, H.; Nalwa, H. S.; Oikawa, H.; Okada, S.; Matsuda, H.; Minami, N.; Kakuta, A.; Ono, K.; Mukoh, A.; Nakanishi, H., Jpn. J. Appl. Phys. 1992, 31, 1132.]. Typically, $16.7 \mu \mathrm{L}$ of a saturated acetone solution of DPCP $(3.3 \mathrm{mg}, 5.51 \mu \mathrm{M})$ was injected into $3 \mathrm{~mL}$ of a vortexing solution of pure $\mathrm{H}_{2} \mathrm{O}$, or $\mathrm{H}_{2} \mathrm{O}$ and surfactant. Independently, $21.0 \mu \mathrm{L}$ of a saturated acetone solution of DCK (4.4 mg, $\left.5.51 \mu \mathrm{M}\right)$ was injected into $3 \mathrm{~mL}$ of a vortexing solution of pure $\mathrm{H}_{2} \mathrm{O}$, or $\mathrm{H}_{2} \mathrm{O}$ and surfactant. For a given single experiment, 10 samples of DCK and DPCP were made, and combined into larger test tubes as early experiments had shown that larger suspensions produced lower quality nanocrystals, i.e. larger particle sizes and size distributions. The larger test tubes were then exposed to reduced pressure for 15 minutes to help remove acetone, then they were sonicated for 30 minutes immediately prior to irradiation. The independently prepared DCK and DPCP suspensions were combined into a $50 \mathrm{~mL}$ Pyrex Erlenmeyer flask, and irradiated for 8 minutes. Every minute, a $6 \mathrm{~mL}$ sample was removed (approximately $1 / 10^{\text {th }}$ of the suspension). The suspensions were extracted with deuterated chloroform $(2 \mathrm{~mL})$, washed with brine $(2 \mathrm{X} 2 \mathrm{~mL})$ and dried over magnesium sulfate. ${ }^{1} \mathrm{H}$ NMR was taken immediately to determine the extent DPA formation. The samples were then subjected to gas chromatography to determine the extent of dicumyl formation. These experiments yields were reproduced in triplicate with $\mathrm{CTAB} / \mathrm{H}_{2} \mathrm{O}, \mathrm{SDS} / \mathrm{H}_{2} \mathrm{O}$, and no surfactant. High conversion data was not used to avoid potential problems caused by the absorption of the products. Gas chromatography was not used to detect DPCP conversion, as DPCP decomposes thermally into DPA. 
Table 1. Representative Data Set (312 nm, CTAB).

\begin{tabular}{|c|c|c|c|c|c|c|c|c|c|}
\hline Trial & \multicolumn{3}{|l|}{ A } & \multicolumn{3}{|l|}{$B$} & \multicolumn{3}{|l|}{$\mathrm{C}$} \\
\hline & \multirow{2}{*}{$\begin{array}{l}\text { Time } \\
\text { Min }\end{array}$} & \multicolumn{2}{|c|}{$\%$ Conversion } & \multirow{2}{*}{$\begin{array}{l}\text { Time } \\
\text { Min }\end{array}$} & \multicolumn{2}{|c|}{$\%$ Conversion } & \multirow{2}{*}{$\begin{array}{l}\text { Time } \\
\text { Min }\end{array}$} & \multicolumn{2}{|c|}{$\%$ Conversion } \\
\hline & & DPCP & DCK & & DPCP & DCK & & DPCP & DCK \\
\hline & 0 & 0 & 0 & 0 & 0 & 0 & 0 & 0 & 0 \\
\hline & 1 & 8.8 & 0.08 & 1 & 15.1 & 0.15 & 1 & 9.7 & 0.14 \\
\hline & 2 & 21.5 & 0.25 & 2 & 29.8 & 0.26 & 2 & 16.7 & 0.26 \\
\hline & 3 & 24.9 & 0.5 & & & & 3 & 32.3 & 0.43 \\
\hline & 4 & 36.6 & 0.7 & 4 & 58.1 & 0.55 & 4 & 56.8 & 0.79 \\
\hline & 5 & 46.4 & 0.98 & 5 & 68.4 & 0.8 & 5 & 67.9 & 1.08 \\
\hline & 6 & 55.1 & 1.2 & 6 & 79.2 & 1.32 & 6 & 74.7 & 1.3 \\
\hline & 7 & 70.5 & 1.3 & 7 & 87.8 & 1.97 & 7 & & \\
\hline \multirow{3}{*}{\multicolumn{2}{|c|}{ Standard Reviation }} & \multicolumn{2}{|l|}{3.18} & \multicolumn{3}{|l|}{3.89} & \multicolumn{3}{|l|}{3.96} \\
\hline & & 3.67 & & & & & & & \\
\hline & & 0.40 & & & & & & & \\
\hline
\end{tabular}

\section{4) Relative Absorbances of DPCP and DCK as a Function of Loading.}

Although each optically dense suspensions can be shown to absorb all of the photons from an immersed light source, ${ }^{1}$ a set of experiments was designed to confirm that the relative absorbance of DPCP and DCK are proportional to their loading in the optically dense mixed suspension. Keeping the total amount of DPCP constant, we varied the amount of DCK added to the suspension to change the DPCP/DCK loading from 0.3 to 3.0. Indeed, the relative conversion of DPCP to DPA and DCK to DC is a linear function of the DPCP/DCK loading ratio.

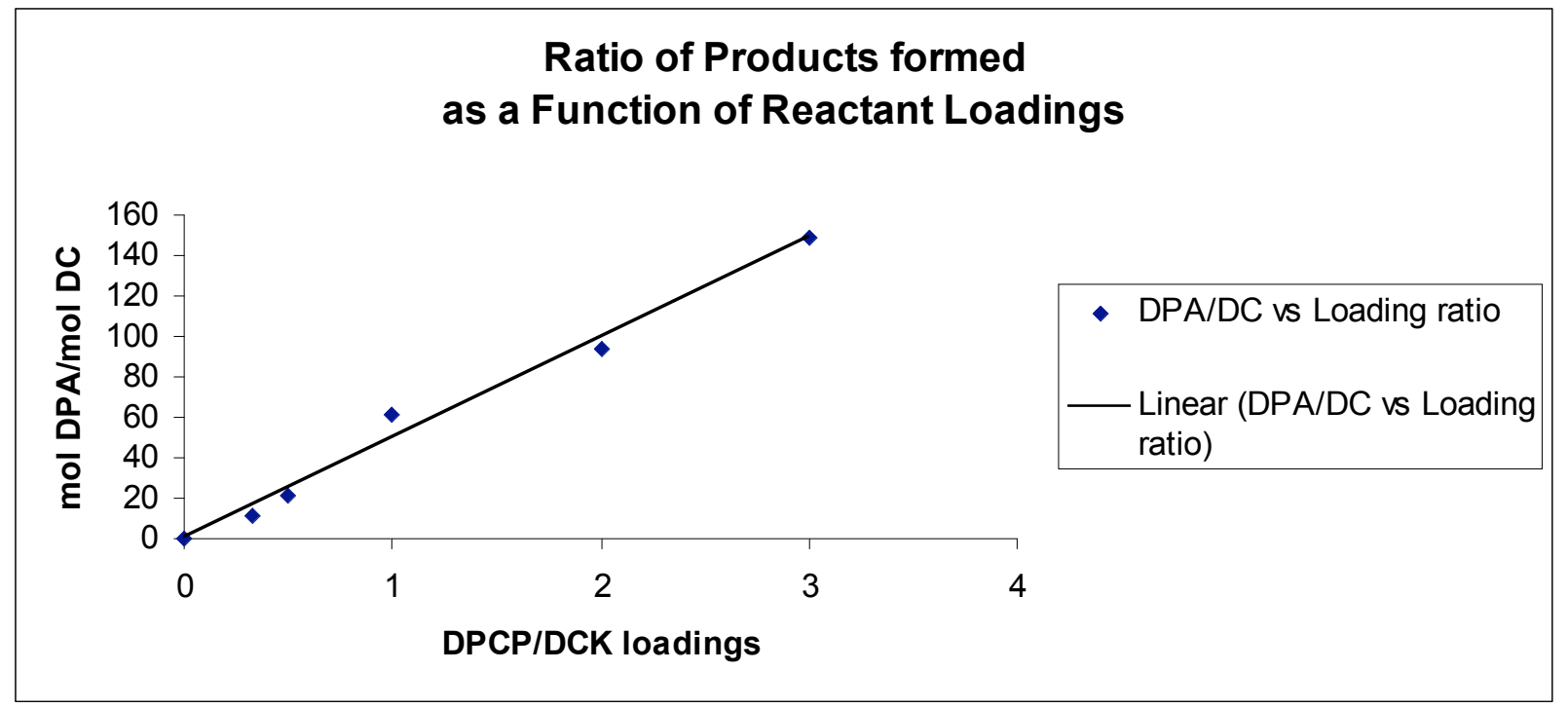

Figure S2. Ratio of products from DPCP and DCK formed as a function of DPCP/DCK loading ratio. 


\section{5) Qualitative Analysis of the Reaction Efficiency as a Function of Excitation to $S_{1}$ and $S_{2}$.}

The purpose of this experiment was to qualitatively document variations in quantum yield of nanocrystalline DPCP as a function of excitation wavelength relative to those of an optically dense solution. We assume that a quantum chain process is more likely in the solid state than in solution, and we interpret an increase at shorter wavelengths in the former as an indication of a quantum chain by excitation to $S_{2}$. Suspensions of DPCP were prepared in the same manner as for the quantum yield study. Solutions of optically dense DPCP in benzene charged with the same amount as the suspensions $(3.3 \mathrm{mg}, 5.36 \mu \mathrm{M})$ were degassed in a septum-sealed test tube for 20 min immediately prior to irradiation. The suspensions and solutions of DPCP were irradiated concurrently in adjacent Pyrex tubes behind six different cut-off filters. Samples were analyzed after two, four, and six minutes intervals. The cut-off filters used were $305,315,338,345,357$, and $366 \mathrm{~nm}$. The suspensions were extracted with deuterated chloroform $(2 \mathrm{~mL})$, washed with brine $(2 \mathrm{X} 2 \mathrm{~mL})$ and dried over magnesium sulfate. ${ }^{1} \mathrm{H}$ NMR of solution and suspension DPCP were taken immediately.

Table 2. Representative Data Set $(\lambda>345 \mathrm{~nm}, \mathrm{CTAB})$

\begin{tabular}{|c|c|c|c|c|c|c|c|c|c|}
\hline \multirow[t]{6}{*}{ Trial } & \multirow{2}{*}{$\begin{array}{l}\text { A } \\
\text { Time } \\
\text { (Min) } \\
\end{array}$} & \multicolumn{2}{|c|}{ DPCP \% Conversion } & \multirow{2}{*}{$\begin{array}{l}\text { B } \\
\text { Time } \\
\text { (Min) } \\
\end{array}$} & \multicolumn{2}{|c|}{ DPCP \% Conversion } & \multirow{2}{*}{$\begin{array}{l}\text { C } \\
\text { Time } \\
\text { (Min) } \\
\end{array}$} & \multicolumn{2}{|c|}{ DPCP \% Conversion } \\
\hline & & Suspension & Solution & & Suspension & Solution & & Suspension & Solution \\
\hline & 0 & 0 & 0 & 0 & 0 & 0 & 0 & 0 & 0 \\
\hline & 2 & 2.9 & 3 & 2 & 3.5 & 4.2 & 2 & 2.1 & 3.1 \\
\hline & 4 & 17.9 & 12.5 & 4 & 15.6 & 12.1 & 4 & 8.3 & 6.3 \\
\hline & 6 & 33.9 & 29.6 & 6 & 21.4 & 17.3 & 6 & 16.5 & 12.2 \\
\hline \multirow{2}{*}{\multicolumn{2}{|c|}{$\begin{array}{c}\Phi_{\text {susp }} / \Phi_{\text {sol }} \text { (relative) } \\
\text { Average } \\
\text { Standard Deviation }\end{array}$}} & \multicolumn{2}{|l|}{1.2} & \multicolumn{3}{|l|}{1.23} & \multicolumn{3}{|l|}{1.3} \\
\hline & & $\begin{array}{r}1.24 \\
\pm 0.037\end{array}$ & & & & & & & \\
\hline
\end{tabular}

Table 3. Summary of wavelength the dependence of relative quantum yields in suspension and in solution

\begin{tabular}{|c|c|}
\hline $\boldsymbol{\lambda} \mathbf{( n m )}$ & $\Phi_{\text {susp }} / \Phi_{\text {sol }}$ \\
\hline 305 & 1.66 \\
\hline 315 & 1.67 \\
\hline 338 & 1.25 \\
\hline 345 & 1.24 \\
\hline 357 & 1.21 \\
\hline 366 & 0.94 \\
\hline
\end{tabular}

\section{6) Experimental Evidence of a Solid-to-Solid Reaction by Fluorescence Analysis.}


DPCP showed no measurable emission spectra. In contrast, DPA samples in $10^{-6} \mathrm{M}$ concentrations in spectral grade n-hexane, as a bulk solid, as a nanocrystalline suspension in $\mathrm{CTAB} / \mathrm{H}_{2} \mathrm{O}$, or in a micellar solution. Also, because $\mathrm{CTAB} / \mathrm{H}_{2} \mathrm{O}$ was emissive, they were carefully analyzed. The emission spectra measured in solution and in micelles are essentially identical. The fluorescence excitation and emission spectra in solution have $\lambda_{\max }=292 \mathrm{~nm}$ and $310 \mathrm{~nm}$, in agreement with literature reports [Fenate, C.; Kensy, U.; Dick, B., J. Phys. Chem. 1993, 97, 13457.]. Similarly, the emission spectra measured from bulk solids and from nanocrystalline suspensions matched each other well. The fluorescence excitation and emission spectra of the solid samples is red shifted to $\lambda_{\max }=335 \mathrm{~nm}$ and $378 \mathrm{~nm}$, respectively. Fluorescence analysis of a photolyzed nanocrystalline suspension of DPCP produced an emission spectrum identical to that obtained from solid DPA.
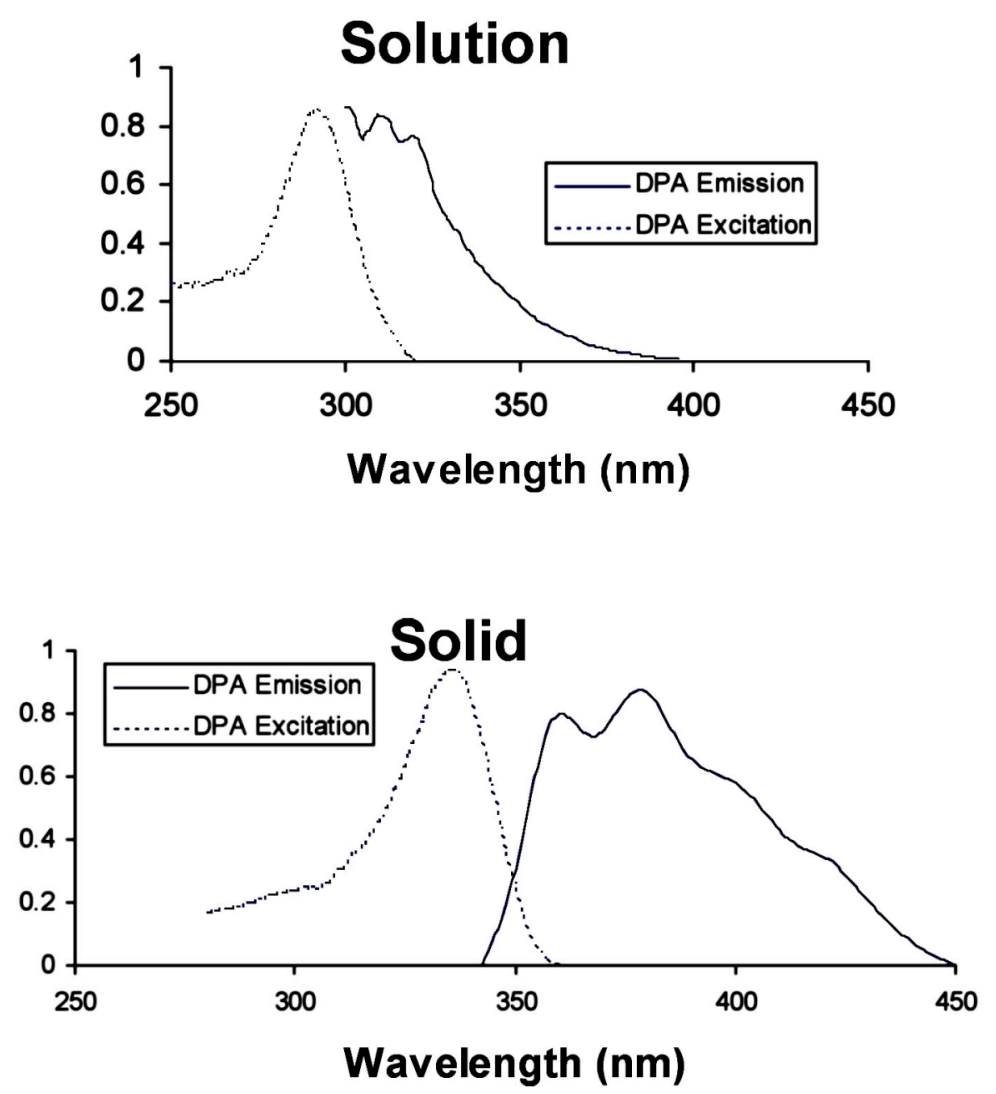

Figure S3. Fluorescence excitation and emission spectra of DPA (diphenyl acetylene) in solution (top), and obtained from photolysis of DPCP in a nanocrystalline suspension (bottom). The bottom spectra are identical to those obtained from commercial DPA as a nanocrystalline suspension or as a bulk solid.

7) Experimental Analysis of Changes in Nanocrystal Size as a Function of Reaction Progress. 
Optically dense suspensions of DPCP were made by injecting $16.7 \mu \mathrm{L}$ of a saturated acetone solution of DPCP $(3.3 \mathrm{mg}, 5.36 \mu \mathrm{M})$ into $3 \mathrm{~mL}$ of a vortexing solution of $\mathrm{H}_{2} \mathrm{O}$ and surfactant. The test tubes were then submitted to reduced pressure for 15 minutes to ensure all acetone was removed. These were then combined and sonicated for 30 minutes prior to irradiation. During irradiation, $3 \mathrm{~mL}$ aliquots were removed at 0 , 4, and 10 minute intervals. The aliquots were then directly analyzed by dynamic light scattering. Afterwards, the suspensions were extracted with deuterated chloroform $(2 \mathrm{~mL})$, washed with brine $(2 \mathrm{X} 2 \mathrm{~mL})$ and dried over magnesium sulfate. ${ }^{1} \mathrm{H}$ NMR was taken immediately to determine the extent of reaction for all of the samples. The average particle size and average percent completion were used as data points to determine the size vs. percent completion. This phenomenon was confirmed in triplicate.

\begin{tabular}{|c|c|c|c|c|c|c|c|}
\hline Ungle & $\begin{array}{c}\text { Mean } \\
(\mathbf{n m})\end{array}$ & $\begin{array}{c}\text { Std.Dov } \\
(\mathbf{n m})\end{array}$ & $\begin{array}{c}\text { Baseline } \\
\text { Error }\end{array}$ & P.I. & Counts/s & $\begin{array}{c}\text { Diff.Coef } \\
\left(\mathbf{m}^{2} / \mathbf{s}\right)\end{array}$ & Overflow \\
\hline $90.0^{\circ}$ & 1084.4 & 424.6 & $0.01 \%$ & 0.198 & $6.23 \mathrm{e}+06$ & $4.17 \mathrm{e}-13$ & 0 \\
\hline
\end{tabular}

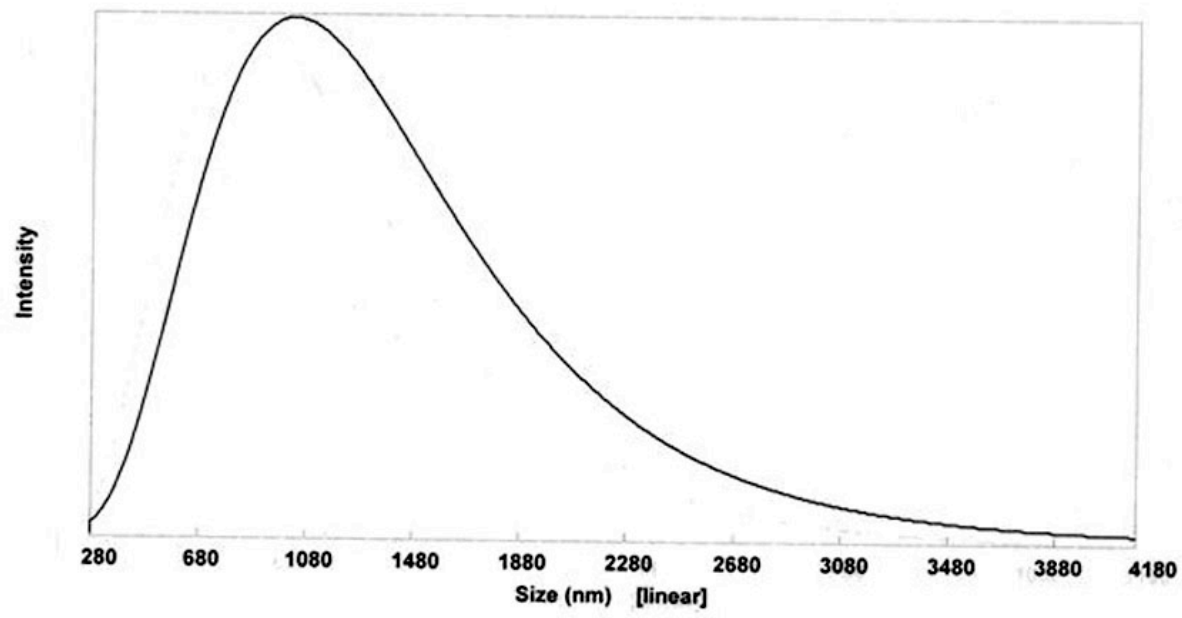

Figure S4. Representative particle size distribution of a sample before irradiation at determined by dynamic light scattering. 


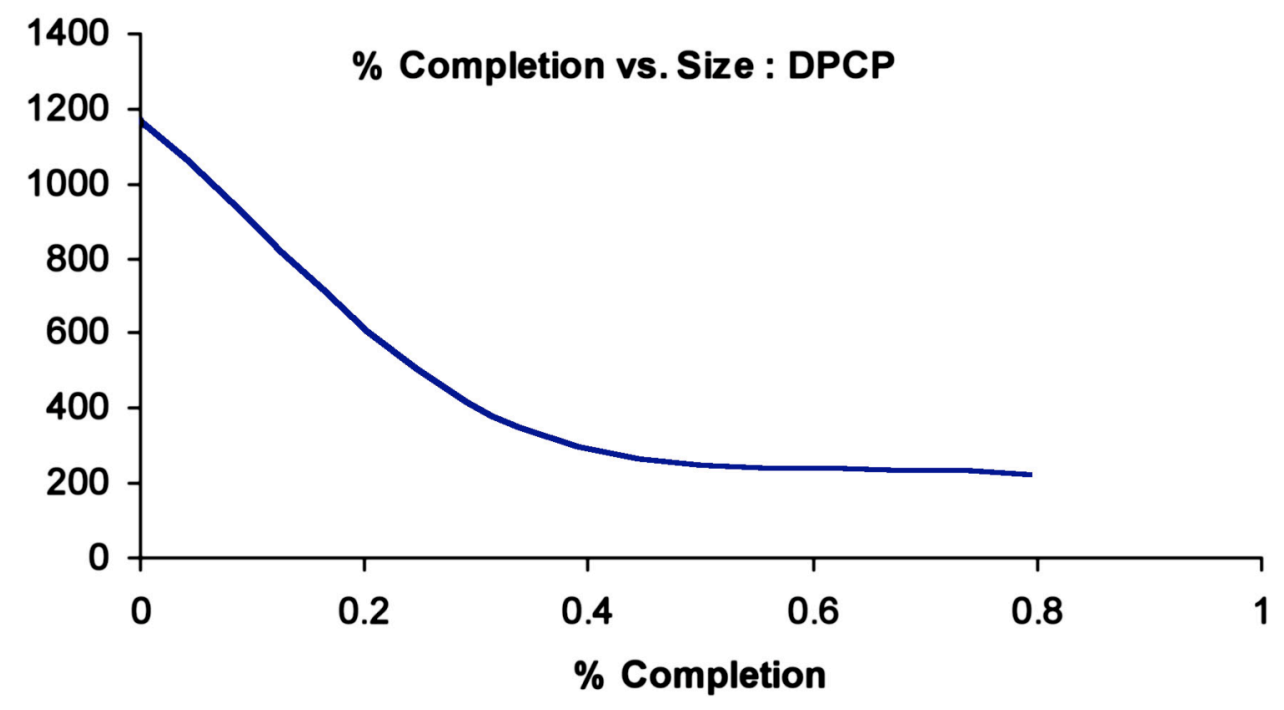

Figure S5. Changes in average particle size as a function of reaction progress for nanocrystalline suspensions of DPCP transforming into DPA.

\section{8) Infrared Spectral Analysis of "As Formed" and recrystallized DPA}

Infrared spectra of commercial DPA, and from DPA generated by photolysis of DPCP were analyzed to determine whether or not they belong to the same crystal form. The spectra from nanocrystalline DPA was obtained with crystals obtained by centrifugation, washed with distilled water, and dried under reduced pressure. The identity of the two spectra suggests that DPA is formed in the same phase as crystals formed under equilibrium in solution.

\section{9) Details on the Accompanying Time-Lapse Microphotographs.}

Microphotographs of several single crystals were obtained every ca. $14.2 \mathrm{~s}$ while irradiating for $20 \mathrm{~min}$ with the output of a medium Presure Hg arc Oriel lamp passed through a Pyrex glass filter.

The files are QuickTime ${ }^{\mathrm{TM}}$ Version 7.2.0, Player Version 7.2 (7.2)

DPCP_hydrate.mov : This illustrates single crystals of the DPCP monohydrate transforming into DPA in a solid-to-solid reconstructive transformation. The first frame was recorded after several seconds. The sample in the last frame was shown to be $100 \%$ conversion.

DPCP_hydrate.mov : This experiment illustrates the evolution of a polycrystalline (powder) sample of anhydrous DPCP transforming into DPA in a solid-to-liquid-to-solid transformation. 


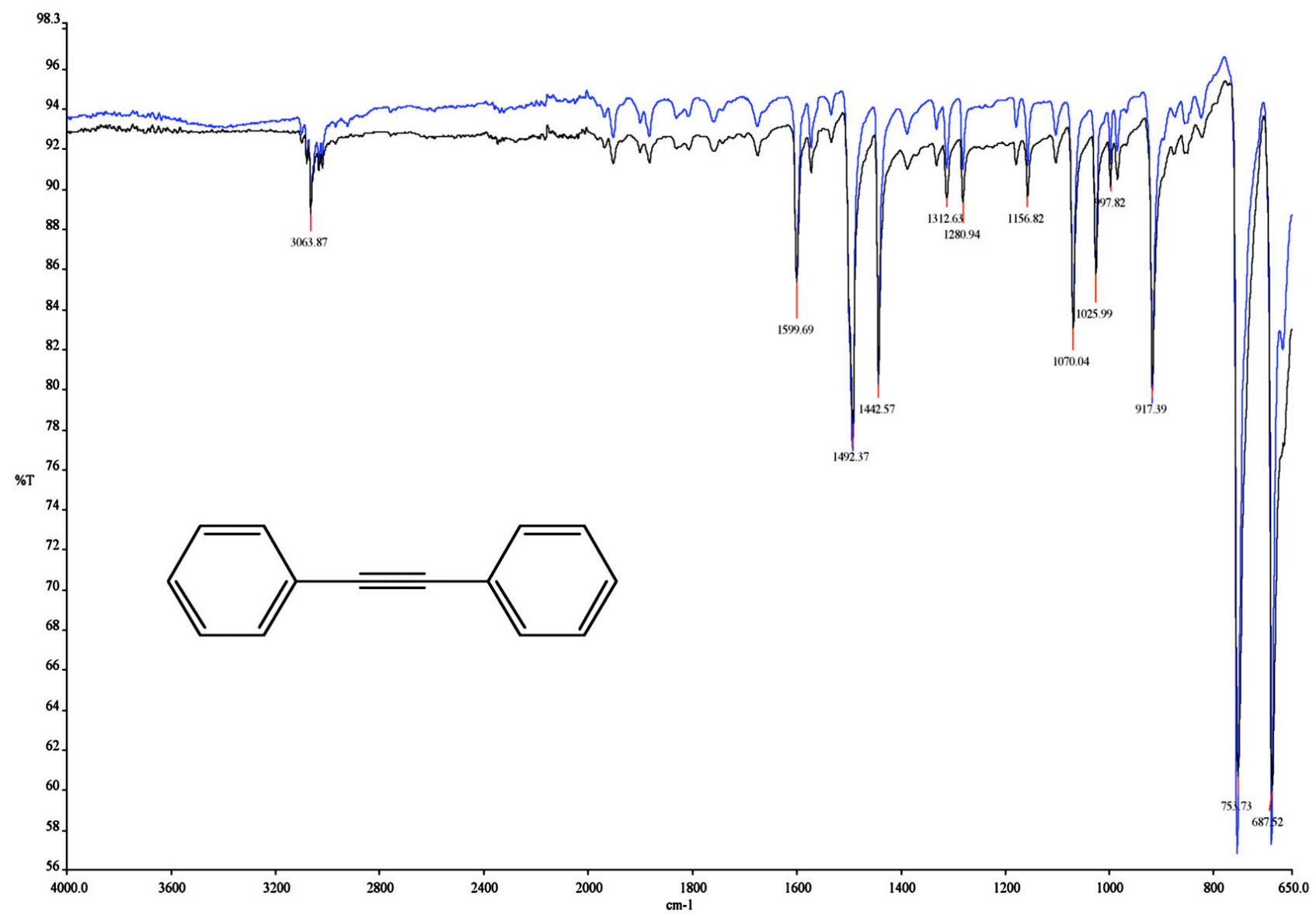

Figure S6. FT-IR spectra of diphenyl acetylene recrystallized from cyclohexane (upper spectrum in blue color) and from centrifuged nanocrystals (lower spectrum in black). The excellent match between the two spectra is interpreted as an indication that the two samples crystallize in the same crystal form. 